\title{
Taurine in Lower Concentration Attenuates Platelet Activity
}

\author{
A. B. Santhakumar', M. D. Linden ${ }^{2}$, I. Singh ${ }^{1, *}$ \\ ${ }^{1}$ School of Medical Science, Gold Coast Campus, Griffith University, Queensland, 4222, Australia \\ ${ }^{2}$ School of Medical Science, RMIT University, Bundoora, Victoria, 3083, Australia
}

\begin{abstract}
Taurine, 2-aminoethanesulphonic acid is a common ingredient of energy drinks which is very popular with young adults. Taurine in energy drinks is known to enhance muscular performance in athletes. However, caffeine in high concentrations as found in most energy drinks have also been implicated to play an adverse role leading to cardiovascular diseases (CVD). Hyper activation of platelets is one of the major risk factors of CVD. The aim of the study was to evaluate effect of taurine alone on platelet aggregation and activation of platelet surface antigens by flowcytometry. It is hypothesized that taurine's antioxidant property would inhibit platelet activity. Twelve healthy, male and female, volunteers aged 20-60 years were recruited for this study. A statistically significant inhibition of platelet aggregation was observed upon stimulation with agonists adenosine diphosphate (ADP), collagen and arachidonic acid (AA) $(p<0.05)$. The platelet surface expression of PAC1 and p-selectin induced by all three agonists also decreased compared to baseline but was not statistically significant. In conclusion this study demonstrated that taurine at a concentration of $500 \mu \mathrm{M}$ (energy drinks contain approximately $31 \mathrm{mM}$ taurine), inhibits the platelet hyperactivity in platelet rich plasma. Further mechanistic and interventional studies are required to examine the pathway involved in support of beneficial role of taurine in prevention of risk factors leading to cardiovascular disorders. Synergistic effect of varying doses of caffeine and taurine will also be evaluated to obtain most effective and protective doses for energy drinks.
\end{abstract}

Keywords Energy Drinks, Taurine, Platelet, Cardio Vascular Disease (CVD)

\section{Introduction}

Taurine is commonly found in very high concentrations in energy drinks. Meat and fish are the major dietary sources of taurine[1, 2]. Energy drinks are becoming increasingly popular with young sports people and are marketed as performance enhancers. Energy drinks commonly contain about $1000 \mathrm{mg}$ taurine, $80 \mathrm{mg}$ caffeine and $600 \mathrm{mg}$ of glucouronolactone[3]. Exercise causes a significant reduction of taurine levels in skeletal muscle. Energy drinks are expected to increase the endurance performance in humans and it has been stated that a significant portion of these effects is due to taurine alone[4-6]. However, several studies have been conducted emphasizing the deleterious effects of such energy drinks on cardiac health. Worthley et al have shown that energy drink consumption acutely increases platelet aggregation and decreases endothelial function in young adults[3]. The limitation of most studies has beenthat they failed to determine which particular component of the energy drink was causing increased platelet aggregation

* Corresponding author:

i.singh@griffith.edu.au (I. Singh)

Published online at http://journal.sapub.org/fph

Copyright (C) 2012 Scientific \& Academic Publishing. All Rights Reserved and endothelial dysfunction. Eli et al observed the inhibition of platelet aggregation by caffeine due to an increase in cyclic adenosine monophosphate (cAMP)[7]. Our current study investigated the effect of taurine on platelet function as there has been limited work done on the effect of taurine alone.

Taurine is abundant in tissues of the brain, heart, retina and platelets. About $20 \mathrm{mM}$ or higher concentrations of taurine may be found in the heart[8]. Synthesis of taurine occurs in the pancreas via the cysteine sulfinic acid pathway [9]. Cysteine dioxygenase oxidizes the sulfhydryl group of cysteine to its acid form, which is then decarboxylated to form hypotaurine. Some of the metabolic roles of taurine include modulation of cellular calcium levels, membrane stabilization, bile acid conjugation and osmoregulation [9-11].

Taurine supplements are being increasingly used for the treatment of congestive heart failure, hypercholesterolemia, hepatitis, high blood pressure, diabetes, disorders of the retina and epilepsy[12-15]. It has also been shown to be effective as an antioxidant and thus protecting against toxicity of various substances such as lead and cadmium [16-19]. It has been demonstrated that taurine reduces the concentrations of serum lipids and apolipoproteins, in hu- 
man liver model HepG2 cells, which are known precursors of coronary heart diseases and arterial thrombosis[20].

Blood platelets play a critical role in haemostasis and thrombosis. Normally, a delicate balance between the interaction of platelets, coagulation factors and the fibrinolytic system maintains haemostatic integrity[21]. It is not clear if taurine activates or inhibits platelet activity. Effect of taurine on platelet function could possibly attenuate risk of thrombosis or may lead to disruption in haemostasis. Platelets generally are found in the circulation in a quiescent state and are anucleated cells. Platelets do have dense granules and alpha granules, which release their content upon platelet activation and initiate the coagulation cascade. Activated platelets help to arrest bleeding as a result of endothelial damage via the coagulation cascade. Activated platelets along with the localized platelet activating factors help to form a stable haemostatic plug[21]. Some of these platelet factors include agonists such as ADP, thromboxane (TXA2), serotonin, collagen, thrombin, AA, epinephrine and nor-epinephrine. Regulatory factors such as plasminogen activator inhibitor (PAI1), tissue plasminogen activator inhibitor (tPAI) are substances that inhibit platelet aggregation[22-24].

Activation of platelets with ADP occurs via P2Y1 (mediates platelet shape change) and P2Y12 (stabilization of clot), which are the two receptors on the platelet surface. ADP in concentrations of $5 \mu \mathrm{mol} / \mathrm{L}$ or higher is responsible for platelet aggregation directly that is independent of the release of platelet granule-contained ADP. Standardization of the optimal concentration of the agonist to be added to the suspension to cause aggregation is an important consideration for any study[22-26]. Thromboxane receptors TP $\alpha$ and TP $\beta$ are responsible for Thromboxane induced activation. Cyclooxygenase -1 (COX-1) and thromboxane synthase are the enzymes, which convert AA to TXA2. AA is a fatty acid found in the granules and membranes of platelets and helps to observe the integrity of the COX pathway. TXA2 similar to ADP helps in the enhancement of platelet aggregation and formation of primary platelet plug[27]. Collagen is considered to be a strong thrombogenic substrate. Platelet surface glycoproteins GP Ib $\alpha$ and GP IIb/IIIa interact with collagen indirectly via von Willebrand Factor (vWF). Under high shear collagen binds to GP Ib $\alpha$ and leads to platelet adhesion, in the presence of vWF. GP VI receptor facilitates platelet activation, which is essential for adhesion and degranulation of activated platelets by binding to collagen [24].

Platelet activation markers are activation dependant monoclonal antibodies (MAb), which assess the conformational changes in the GPIIb-IIIa complex and granule membrane proteins (integrin $\alpha_{\mathrm{II}} \beta_{3}$ ). The receptor for vWF and fibrinogen responsible for platelet aggregation is integrin $\alpha_{\mathrm{II}} \beta_{3}$. Antibody PAC1 is directed against the fibrinogen-binding site when a conformational change in $\alpha_{\mathrm{IIb}} \beta_{3}$, due to platelet activation, is encountered. It binds only to activated platelets and not resting platelets. A few monoclonal antibodies such as CD41 or CD61 bind to rest- ing platelets. Antibodies p-selectin or CD62P are directed against the granule membrane proteins, which are, expressed only when the $\alpha$-granules are released and does not bind to resting platelets. Thrombin receptor-activating peptide (TRAP) is a peptide fragment, serine protease of the protease- activated receptor 1 (PAR1). TRAP directly helps in activating platelets without resulting in a fibrin clot. TRAP acts very similar to thrombin by not causing proteolyic cleavage making it a suitable agonist for observation of thrombin induced platelet activation[27, 28].

Miglis et al (2002) provided data in support of the inhibitory effect of taurine on platelets and the plasma coagulation system. A final concentration of $5 \mathrm{mM}$ taurine was used in the above study, which inhibited platelet aggregation by $10 \%[29]$. The aim of the current study is to evaluate the effect of lower concentration of taurine on platelet activity. This aim is to prove the hypothesis that beneficial impact of Taurine on cardiovascular health may be result of its inhibitory effect on platelet hyperactivity, which may be instrumental in preventing thrombotic risk factors. After completing a pilot study of dose response curve, a lower a concentration of taurine $(500 \mu \mathrm{M})$ was selected for this study. Hence, the overall aim of this study was firstly to determine the least dose of taurine that may lead to inhibition of platelet aggregation in-vitro, in response to stimulation with ADP, collagen and AA; Secondly, to evaluate the effect of taurine on platelet hyperactivation or inhibition of activity by measuring platelet surface antigen expression using flow cytometry in addition to platelet aggregation studies.

\section{Materials and Methods}

This study was performed at the School of Medical Sciences, RMIT University, Melbourne, Australia. The RMIT Human Research Ethics Committee, RMIT University, Melbourne, Australia, approved this study.

\subsection{Subjects and Blood Collection}

Twelve healthy volunteers aged 20-60 years were recruited for this study. The mean age of volunteers who participated in this study was $31 \pm 8$ (8 males \& 8 females) for platelet aggregometry and $32 \pm 10$ ( 6 males $\& 6$ females) for flowcytometry. $10 \mathrm{~mL}$ of venous blood was collected from volunteers on two separate occasions. Blood was collected in, $2 \mathrm{~mL}$ tri-potassium ethylenediaminetetraacetic acid (EDTA-1.8mg/mL) for Full Blood Examination (FBE) and two- $5 \mathrm{~mL} 3$ tri-sodium citrate $(3.8 \%)$ tubes for platelet aggregometry and flowcytometry. Volunteers completed questionnaires and were not included in the study if they were taking non-steroidal inflammatory drugs, aspirin-type products, energy drinks, and dietary antioxidant supplements for at least one week prior to study commencement. Participants were requested and voluntarily agreed not to consume alcoholic beverages 2 days prior and caffeine for at least 24 hours prior to the study. For this in-vitro study 
compliance was only confirmed by the volunteer's statement on the questionnaire before they signed informed consent for phlebotomy.

\subsection{Full Blood Examination (FBE)}

Whole blood collected in EDTA tubes were used to determine the platelet count and mean platelet volume (MPV) within 10-15 minutes of venepuncture. The instrument used for the analysis was the Beckman Coulter AcTTM 5Diff CP hematology analyzer (Coulter Corporation, Miami, Florida, USA). The analyzer was pre-calibrated with the AcTTM 5Diff Cal Calibrator (Beckman CoulterTM, Miami, Florida, USA). Volunteers with platelet count within the reference range of $(150-400) \times 10^{9} / \mathrm{L}$ and MPV of $6-10 \mathrm{fL}$ were included in the testing process.

\subsection{Taurine Working Standard}

$500 \mu \mathrm{M}$ concentration of taurine working solution was used for the study. First, $250 \mathrm{mM}$ stock solution of taurine was prepared by dissolving $31.25 \mathrm{gms}$ of commercially available taurine (ICN Biomedicals Inc, Ohio) in $1 \mathrm{~mL}$ of phosphate buffer saline (PBS) with a physiologic $\mathrm{pH}$ of 7-7.5. Then, $500 \mu \mathrm{M}$ of taurine was prepared from the stock solution by using the serial dilution method. Prior to use, stock and working solutions of Taurine and PBS were aliquoted and stored in the freezer at $-4^{\circ} \mathrm{C}$ to avoid bacterial contamination.

\subsection{Platelet Aggregometry}

PRP was tested with and without in-vitro addition of $500 \mu \mathrm{M}$ taurine. Platelet aggregation was carried out in PRP using three different agonists - ADP $(10 \mu \mathrm{M})$, collagen $(2.4 \mu \mathrm{g} / \mathrm{mL})$, and AA $(0.3 \mathrm{mM})$. Aggregation testing was carried out using a dual channel Lumi- aggregometer Model- 700 (Chrono-Log Corporation, Philadelphia, USA). The aggregometer was equipped with Chrono-Log AGGRO/LINK ${ }^{\circledR} 8$ (Version 1.2.2) interface for data transfer into a Microsoft Windows ${ }^{\circledR}$ compatible computer. $500 \mu \mathrm{M}$ taurine was added to the test blood in cuvettes (Chrono-Log Corporation, Philadelphia, PA, USA) and incubated at $37^{\circ} \mathrm{C}$ for one hour (prior to study various incubation periods were trialed and $60 \mathrm{~min}$ was found to be most effective, data not provided here). The samples were then placed inside the heating block testing wells of the aggregometer for $2 \mathrm{~min}$ at $37^{\circ} \mathrm{C}$ to ensure stability before analysis. Siliconised stir bars (Chrono-Log Corporation, Philadelphia, PA, USA) were then added to the cuvettes to facilitate thorough mixing of the sample. The respective agonist was added to the sample and aggregation chart recording initiated. The platelet aggregation was recorded for 6 minutes. The amplitude of platelet aggregation and the slope were recorded.

\subsection{Flow Cytometry}

Flow Cytometry on whole blood obtained from participants were analyzed using a BD FACSCanto II cytometer (BD Biosciences, USA) and the data obtained from the testing was recorded and interpreted using BD FACS DivaTM software (BD Biosciences, USA). The first step involved was the collection of blood from the participants into a $5 \mathrm{~mL} 3$ tri-sodium citrate (3.8\%). After 1-hour incubation of blood sample with $500 \mu \mathrm{M}$ taurine, a cocktail mix (Table 1) of an isotype control or the test antibody were added to respective control and test sample tubes. Agonists were added to the suspension containing the blood and the cocktail mix. All the samples for flowcytometric analysis were fixed with $1 \%$ formaldehyde since it was not feasible to analyze the samples within a short period. The suspension was then analyzed in the BD FACSCanto II cytometer.

Table 1. Composition of Isotype Control and Test antibody cocktail

\begin{tabular}{cc}
\hline Isotype Control & Volume $(\mu \mathrm{L})$ \\
\hline PAC1-FITC & 75 \\
$200 \mu \mathrm{g} / \mathrm{mL}$ Eptifibatide & 7.5 \\
$1 \mu \mathrm{g} / \mathrm{mL}$ MIgG1-PE & 12 \\
CD 42b-PCy-5 & 50 \\
HS-BSA-Azide & 155.5 \\
Total volume & 300 \\
\hline Test Antibody & \\
\hline PAC1-FITC & 225 \\
CD62P-PE & 288 \\
CD 42b-PCy-5 & 150 \\
HS-BSA-Azide & 237 \\
Total volume & 900 \\
\hline
\end{tabular}

FITC- Fluorescein isothiocyanate, HS- Hepes saline, BSA- Bovine serum albumin. FITC is used along with PAC1 to help in fluorescing activated platelets. PCy-5 used with CD $42 \mathrm{~b}$, binds to resting platelets. The monoclonal mouse IgG1, kappa immunoglobulin is used as an isotype control.

\subsection{Power Calculations and Statistical Methods}

A final sample size of 12 volunteers in each group is required for $80 \%$ power to detect a $5 \%$ variation in the laboratory parameters measured, where a 3-5\% standard deviation exists in the population, assuming an alpha error of 0.05 . The effect of taurine on platelet aggregation and activation was compared by paired t-tests. The data was analyzed for significant values $(\mathrm{P}<0.05)$. The above test was performed using the Graph pad Prism ${ }^{\circledR}$. For flowcytometric data analysis, a two way analysis of variance (ANOVA) was employed to calculate the mean fluorescence intensity (MnIX) and percentage activation for platelets. This test was performed using IBM SPSS Statistics $18^{\circledR}$ software.

\section{Results}

\subsection{Subjects}

Results from the FBE showed that the 12 subjects had a mean platelet count of $255 \times 10^{9} / \mathrm{L}$ and MPV of $8.36 \mathrm{fL}$, used for platelet aggregometric studies and $243 \times 10^{9} / \mathrm{L}$ and MPV of $8.52 \mathrm{fL}$ for flowcytometry. The recommended healthy 
reference ranges for PLT and MPV are $150-400 \times 10^{9} / \mathrm{L}$ cells and 6-10fL respectively.

\subsection{Effect of Taurine on Platelet Aggregation}

Figure 1 shows the percentage change in the aggregation from the baseline $(100 \%$ aggregation), post taurine treatment, using all three agonists. Table 2 shows the comparison of mean percentage aggregation, Standard deviation between control and taurine treated samples.

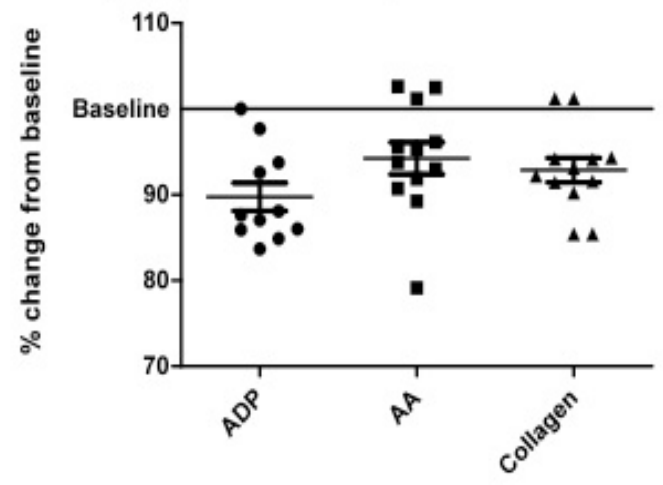

Figure 1. Percentage change in aggregation post Taurine treatment from the baseline value (100\% aggregation). The figure shows a statistically significant reduction in aggregation of platelets by all the agonists $(P<0.05)$.

Table 2. Comparison of Mean percentage aggregation between control \& test samples

\begin{tabular}{|c|c|c|c|c|c|c|}
\hline & \multicolumn{2}{|c|}{ ADP } & \multicolumn{2}{|c|}{$\begin{array}{l}\text { Arachidonic } \\
\text { Acid }\end{array}$} & \multicolumn{2}{|c|}{ Collagen } \\
\hline & $\begin{array}{c}\text { Con- } \\
\text { trol }\end{array}$ & Test & $\begin{array}{c}\text { Con- } \\
\text { trol }\end{array}$ & Test & $\begin{array}{c}\text { Con- } \\
\text { trol }\end{array}$ & Test \\
\hline $\begin{array}{c}\text { Mean \% } \\
\text { Aggrega- } \\
\text { tion }\end{array}$ & 74.75 & 66.16 & 87.25 & 81.91 & 87.66 & 81.25 \\
\hline $\begin{array}{l}\text { Standard } \\
\text { Deviation }\end{array}$ & 16.93 & 17.55 & 7.09 & 4.71 & 5.44 & 3.62 \\
\hline
\end{tabular}

*Statistically significant reduction in platelet aggregation $(\mathrm{P}<0.05)$.

\subsection{Flowcytometry}

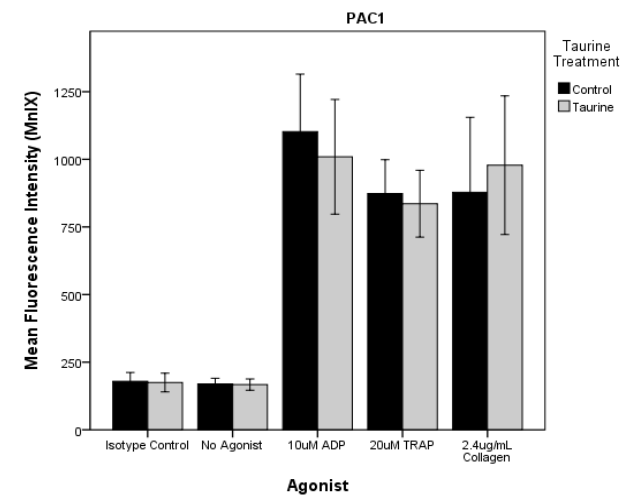

Figure 2. Mean fluorescence intensity expression in PAC1 induced by ADP, TRAP and Collagen. No significant inhibition or activation of platelets after Taurine treatment was observed.

Figures 2 and 3 shows the mean fluorescence intensity expression (MnIX) for PAC1 and p-selectin induced by
ADP, collagen and TRAP. The control sample did not contain any agonist (NA). One of the volunteers exhibited abnormal MnIX expression for PAC1 induced by ADP. Hence it was considered to be a clear outlier and was excluded from the statistical analysis.

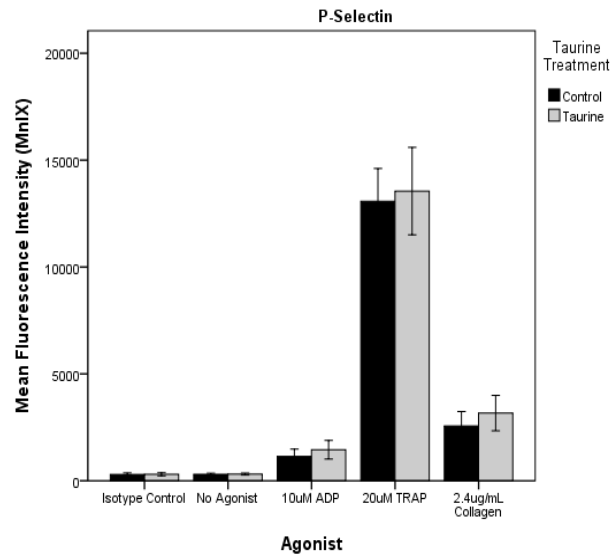

Figure 3. Mean fluorescence intensity expression in P-Selectin induced by ADP, TRAP and Collagen. No significant inhibition or activation of platelets after Taurine treatment was observed.

\section{Discussion}

The aim of the study was to determine the inhibition of platelet activation by taurine using platelet aggregometry and flow cytometry techniques. Results of this study demonstrate a statistically significant inhibition of platelet aggregation in PRP induced by all agonists used including ADP, collagen and AA $(\mathrm{P}<0.05)$. These results demonstrate that taurine may be instrumental in preventing risk factors leading to CVD by inhibiting platelet hyperactivity. Flowcytometric studies using ADP and TRAP as agonist exhibited reduced PAC1 expression in taurine treated samples, but the results were not statistically significant, suggesting there may be minimal effect of taurine on fibrinogen binding site. On the other hand $\mathrm{p}$-selectin expression induced by all the agonists used showed mild activation of the platelets, but were not statistically significant $(\mathrm{P}>0.05)$. This indicates that taurine did not have significant effect on alpha granule release from activated platelets. It was also observed that expression of PAC1 and p-selectin monoclonal antibodies was considerably high when induced by TRAP. A cytosolic form of the enzyme phospholipase A2 has been proven to play a major role in the release of arachidonic acid in platelets[30, 31]. TRAP is known to phosphorylate phospholipase A2 thereby resulting in the stimulation of arachidonic acid. The released arachidonic acid is converted to thromboxane $\mathrm{A} 2$ by the COX pathway thereby further activating the platelets[30]. This may be the reason for the high expression of PAC1 and p-selectin when TRAP was used as the agonist.

Previous studies have shown the inhibition of platelet activation by high concentrations of taurine but did not determine the optimal sub maximal concentration of taurine that 
may cause the similar effect. They had established that a final concentration of $5 \mathrm{mM}$ taurine inhibited platelet aggregation by $10 \%[29]$. In this study, we were able to show similar inhibitory effect using a lower dose of $500 \mu \mathrm{M}$ taurine. Furthermore, they have not analyzed platelet activation using flow cytometry. In this study, the optimal dose was determined by the preliminary testing to analyze platelet function by flowcytometry, in addition to aggregation studies.

The volunteers had to complete a screening form. This questionnaire helped to recruit the participants based on the exclusion criteria included in material and methods section. Since it was not feasible to monitor the dietary compliance, participants completed a second questionnaire at the time of signing consent form for phlebotomy to confirm the dietary compliance. For example, a cup of coffee containing caffeine could influence platelet activation. Even heavy exercise or underlying allergies could alter platelet function. Hence, considering such factors and following stringent rules could have helped in better data acquisition. However this was a pilot in vitro study and variables mentioned above will be taken into consideration and implemented in next intervention study. There seemed to be an increase in the standard deviation in the control and taurine treated samples in platelet aggregation induced by ADP. This was because of two outliers amongst the twelve volunteers. Although the two outliers were included in the statistical analysis, significant reduction in platelet aggregation induced by ADP was observed $(\mathrm{P}<0.05)$.

A reference range of $150-400 \times 10^{9} / \mathrm{L}$ cells and 6.0 to 10.0fL for PLT and MPV were used as part of the exclusion criteria. Blood samples were collected at two different occasions for platelet aggregometry and flow cytometry respectively. Ideally all testing should have been done on the sample collected from same bleed to avoid intrapersonal variation in the results of platelet count and MPV. Changes in these parameters could affect the mean activation or aggregation. In this study the intrapersonal variation was minimal as shown by the FBE results.

A statistically significant inhibition of platelet aggregation was seen in the case of platelet aggregometric studies. These results (Figure 1 and Table 2) are consistent with the findings of Miglis et al, which showed a $10 \%$ inhibition of platelet aggregation by taurine at a final concentration of $5 \mathrm{mM}[29]$. However, Worthley et al proved that energy drinks (comprising of taurine) acutely increased platelet aggregation[3]. Hence this study was performed to add to the evidence of whether taurine inhibits or activates platelets. Our results are consistent with Miglis et al, though with a much lower dose of taurine.

It has been reported that artifactual platelet activation might occur due to longer incubation of MoAbs with WB samples[30]. A spontaneous increase in the activation of platelets, determined by an increase in CD62P and p-selectin binding, occurred in WB samples incubated for more than 10 minutes along with $\operatorname{MoAb}[31]$. In this study, the MoAbs were incubated with WB for 15 minutes. The temperature at which the samples had to be processed was an important factor under consideration. Platelets stored at lower than $20^{\circ} \mathrm{C}$ have been shown to possess increased activation levels than those stored at $37^{\circ} \mathrm{C}[32,33]$. Hence from the above findings it was decided that incubation at $37^{\circ} \mathrm{C}$ would be more appropriate to carry out platelet function testing. This could also be because $37^{\circ} \mathrm{C}$ is a normal physiological human body temperature. It is practical to use this incubation temperature because during intervention studies taurine will be interacting with platelets in the body at $37^{\circ} \mathrm{C}$.

From flowcytometric analysis, it was observed that the taurine incubated sample inhibited platelet activation of the control sample when PAC1 was used as the monoclonal antibody and ADP, TRAP were used as the agonists (Figure 2). But when a paired t-test was performed comparing the means of the mean fluorescence intensities (MnIX) of the controls and taurine treated samples (12 subjects), the values were not statistically significant. Furthermore, comparison of the percentage activation of the control and taurine samples did not exhibit significant results. MnIX was used for data analysis, since the results obtained by comparing the mean fluorescence intensities and percentage activation was correlative. The overall expression of $\mathrm{PAC} 1$ induced by ADP and TRAP did show some inhibition of activation but the value was not statistically significant $(\mathrm{P}>0.05)$. A mild activation of the expression of $\mathrm{p}$-selectin induced by all the three agonists was observed in taurine treated samples (Figure 3). It was again observed that there was no significant difference in the mean fluorescence intensity expression in p-selectin induced by the agonists (Figure 3). Currently in literature there are no studies performed by flowcytometry to evaluate the effect of Taurine on platelets. Hence this is one of the novel investigations.

The results obtained from platelet aggregometry and flow cytometry do not appear to correlate well. The inhibition of platelet aggregation as seen on taurine treated samples was not mirrored by a decrease in the expression of activation dependant antigens on the platelet surface. There are several explanations to these findings. Platelet aggregometry is a functional assay whereas; flow cytometry is an immunologic assay. Flow cytometry measures specific events of platelet activation per second using various MoAbs. But platelet aggregometry on the other hand does not measure the platelet activation per second but the entire cascade from platelet activation to the final aggregation to form a stable platelet plug. An earlier study also suggests that platelet aggregation response need not have to necessarily correlate with platelet antigen expression. In contrast, a significant increase in the expression of p-selectin induced by ADP, TRAP and collagen was observed[34, 35]. Further studies are required to examine the mechanistic pathway that may be involved in attenuating platelet activity.

Optimal concentrations of taurine and agonist to be added to PRP in platelet aggregometry to cause sub maximal inhibition of platelet activity were established. The same concentrations of taurine and agonists were used in flowcy- 
tometric analysis using whole blood. In flowcytometric analysis, either the concentration of taurine required to cause inhibition of platelet activation was low or the concentrations of agonists used were high when WB was used. Furthermore, it was hypothesized from this study that taurine did not affect the fibrinogen receptor conformational change (Figure 2) but instead mildly enhanced the $\alpha$-granule release (Figure 3 ) and expression of CD62P induced by ADP, TRAP and collagen[36]. Constant stirring of the taurine incubated sample by the magnetic stirrer in platelet aggregometry; usage of PRP for aggregometry and WB for flow cytometry may be the few factors responsible for non-consistent results between platelet aggregation and flowcytometry.

\section{Conclusions}

In conclusion, this study demonstrated that taurine inhibits the platelet hyperactivity in platelet rich plasma however in the whole blood some of the platelet surface antigen expression did not show any inhibition. Additional antibodies specific for other platelet surface receptors will be evaluated in future studies. These results suggest there may be different mechanisms and pathways involved in taurine's effect on platelet function as p-selectin expression exhibited mild activation of platelets in this study. Further mechanistic and interventional studies are required to provide further evidence in support of beneficial role of taurine in presence of caffeine to establish if energy drinks with lower concentrations of taurine and caffeine may play a role in prevention of risk factors leading to cardiovascular disorders.

\section{ACKNOWLEDGEMENTS}

We gratefully acknowledge the assistance provided by Teresa DeFrancesco and Heather Darmody for their support and help in equipment and facility maintenance. We would also like to thank Lazarela Vucinic and Nicolette Fozzard for their assistance with statistical calculations. We would like to thank all our volunteers for their participation in this study. There were no conflicts of interest.

Authors contributions: MDL \& IS designed research; MDL provided essential reagents; ABS conducted research; ABS \& IS analyzed results; ABS wrote the paper; IS had primary responsibility for final content

\section{REFERENCES}

[1] Bouckenooghe T, Remacle C, Reusens B. Is taurine a functional nutrient? Curr Opin Clin Nutr Metab Care2006 Nov;9(6):728-33.

[2] Brosnan JT, Brosnan ME. The sulfur-containing amino acids: an overview. J Nutr2006 Jun;136(6 Suppl):1636S-40S.

[3] Worthley MI, Prabhu A, De Sciscio P, Schultz C, Sanders P,
Willoughby SR. Detrimental effects of energy drink consumption on platelet and endothelial function. Am J Med2010 Feb;123(2):184-7.

[4] Matsuzaki Y, Miyazaki T, Miyakawa S, Bouscarel B, Ikegami T, Tanaka N. Decreased taurine concentration in skeletal muscles after exercise for various durations. Med Sci Sports Exerc2002 May;34(5):793-7.

[5] Pierno S, De Luca A, Camerino C, Huxtable RJ, Camerino DC. Chronic administration of taurine to aged rats improves the electrical and contractile properties of skeletal muscle fibers. J Pharmacol Exp Ther1998 Sep;286(3):1183-90.

[6] Yatabe Y, Miyakawa S, Miyazaki T, Matsuzaki Y, Ochiai N. Effects of taurine administration in rat skeletal muscles on exercise. J Orthop Sci2003;8(3):415-9.

[7] Lev EI, Arikan ME, Vaduganathan M, Alviar CL, Tellez A, Mathuria N, Builes A, Granada JF, del Conde I, Kleiman NS. Effect of caffeine on platelet inhibition by clopidogrel in healthy subjects and patients with coronary artery disease. Am Heart J2007 Oct;154(4):694 e1-7.

[8] Chesney RW. Taurine: its biological role and clinical implications. Adv Pediatr1985;32:1-42.

[9] Huxtable RJ. Physiological actions of taurine. Physiol Rev1992 Jan;72(1):101-63.

[10] Birdsall TC. Therapeutic applications of taurine. Altern Med Rev1998 Apr;3(2):128-36.

[11] Guizouarn H, Motais R, Garcia-Romeu F, Borgese F. Cell volume regulation: the role of taurine loss in maintaining membrane potential and cell $\mathrm{pH}$. J Physiol2000 Feb 15;523 Pt 1:147-54.

[12] Gordon RE, Park E, Laskin D, Schuller-Levis GB. Taurine protects rat bronchioles from acute ozone exposure: a freeze fracture and electron microscopic study. Exp Lung Res1998 Sep-Oct;24(5):659-74.

[13] Gurujeyalakshmi G, Wang Y, Giri SN. Taurine and niacin block lung injury and fibrosis by down-regulating bleomycin-induced activation of transcription nuclear factor-kappaB in mice. J Pharmacol Exp Ther2000 Apr;293(1):82-90.

[14] Militante JD, Lombardini JB. Taurine: evidence of physiological function in the retina. Nutr Neurosci2002 Apr;5(2):75-90.

[15] Pasantes-Morales H, Quiroz H, Quesada O. Treatment with taurine, diltiazem, and vitamin $\mathrm{E}$ retards the progressive visual field reduction in retinitis pigmentosa: a 3-year follow-up study. Metab Brain Dis2002 Sep;17(3):183-97.

[16] Das J, Ghosh J, Manna P, Sil PC. Taurine provides antioxidant defense against NaF-induced cytotoxicity in murine hepatocytes. Pathophysiology2008 Oct;15(3):181-90.

[17] Green TR, Fellman JH, Eicher AL, Pratt KL. Antioxidant role and subcellular location of hypotaurine and taurine in human neutrophils. Biochim Biophys Acta1991 Jan 23;1073(1):91-7.

[18] Gurer H, Ozgunes H, Saygin E, Ercal N. Antioxidant effect of taurine against lead-induced oxidative stress. Arch Environ Contam Toxicol2001 Nov;41(4):397-402.

[19] Sinha M, Manna P, Sil PC. Taurine protects the antioxidant defense system in the erythrocytes of cadmium treated mice. 
BMB Rep2008 Sep 30;41(9):657-63.

[20] Zhang M, Bi LF, Fang JH, Su XL, Da GL, Kuwamori T, Kagamimori S. Beneficial effects of taurine on serum lipids in overweight or obese non-diabetic subjects. Amino Acids2004 Jun;26(3):267-71.

[21] Hoffbrand AV. Essential Haematology: Platelets, Blood coagulation and haemostasis. third ed: Austraia: Blackwell Science; 1993.

[22] A randomised, blinded, trial of clopidogrel versus aspirin in patients at risk of ischaemic events (CAPRIE). CAPRIE Steering Committee. Lancet. [Clinical Trial Multicenter Study Randomized Controlled Trial Research Support, Non-U.S. Gov't]. 1996 Nov 16;348(9038):1329-39.

[23] Colwell JA, Nesto RW. The platelet in diabetes: focus on prevention of ischemic events. Diabetes Care. [Review]. 2003 Jul;26(7):2181-8.

[24] Hoffbrand AV. Postgraduate Haematology. 5th ed: USA: Blackwell Publishing; 2005.

[25] Devaraj S, Xu DY, Jialal I. C-reactive protein increases plasminogen activator inhibitor-1 expression and activity in human aortic endothelial cells: implications for the metabolic syndrome and atherothrombosis. Circulation. [Research Support, Non-U.S. Gov't Research Support, U.S. Gov't, P.H.S.]. 2003 Jan 28;107(3):398-404.

[26] Dorsam RT, Kunapuli SP. Central role of the P2Y12 receptor in platelet activation. J Clin Invest. [Review]. 2004 Feb;113(3):340-5.

[27] Quin M. FD, Cox D. Platelet Function: Assesment, Diagnosis, and Treatment: USA: Human Press; 2005.

[28] Lepantalo A, Virtanen KS, Resendiz JC, Mikkelsson J, Viiri LE, Karhunen PJ, Lassila R. Antiplatelet effect of clopido- grel in patients with aspirin therapy undergoing percutaneous coronary interventions--limited inhibition of the P2Y12 receptor. Thrombosis research. [Research Support, Non-U.S. Gov't]. 2009 Jun;124(2):193-8.

[29] Miglis M, Wilder D, Reid T, Bakaltcheva I. Effect of taurine on platelets and the plasma coagulation system. Platelets. [Research Support, U.S. Gov't, Non-P.H.S.]. 2002 Feb;13(1):5-10.

[30] AH G. Platelet-asociated molecules and immunoglobulins.: Blackwell Scientific Publications, Oxford; 1994.

[31] Shattil SJ, Cunningham M, Hoxie JA. Detection of activated platelets in whole blood using activation-dependent monoclonal antibodies and flow cytometry. Blood. [Research Support, Non-U.S. Gov't Research Support, U.S. Gov't, P.H.S.]. 1987 Jul;70(1):307-15.

[32] Hansen TN, Brockbank KG. Increased platelet aggregation due to chilling to 20 degrees $\mathrm{C}$ is not related to increased sensitivity to agonists. Transfusion. [Research Support, U.S. Gov't, Non-P.H.S.]. 1997 Jul;37(7):696-702.

[33] Michelson AD. Flow cytometry: a clinical test of platelet function. Blood. [Review]. 1996 Jun 15;87(12):4925-36.

[34] Mutanen M. Cis-unsaturated fatty acids and platelet function. Prostaglandins Leukot Essent Fatty Acids. [Review]. 1997 Oct;57(4-5):403-10.

[35] Serebruany VL, Gurbel PA, Shustov AR, Ohman EM, Topol EJ. Heterogeneity of platelet aggregation and major surface receptor expression in patients with acute myocardial infarction. American heart journal. [Research Support, Non-U.S. Gov't]. 1998 Sep;136(3):398-405.

[36] Michelson AD. Platelets. 2nd ed: USA: Academic Press; 2006. 